\title{
BioXTAS RAW 2.0: The latest in SAXS data analysis
}

\author{
J Hopkins ${ }^{1}$, R Gillilan ${ }^{2}$, S Skou ${ }^{3}$ \\ ${ }^{1}$ BioCAT (Sector 18, APS), Illinois Institute of Technology, Downers Grove, IL, ${ }^{2}$ MacCHESS, Cornell \\ Univ, ${ }^{3}$ Xenocs Nordic, Horsholm, Denmark \\ jhopkins1@iit.edu
}

BioXTAS RAW is a graphical-user-interface-based free open-source Python program for reduction and analysis of small-angle X-ray solution scattering (SAXS) data. RAW 2.0 brings python 3 compatibility, faster integration using pyFAI, and numerous other improvements both small and large. The software is designed for biological SAXS data and enables creation and plotting of one-dimensional scattering profiles from two-dimensional detector images, standard data operations such as averaging and subtraction and analysis of radius of gyration and molecular weight, and advanced analysis such as calculation of inverse Fourier transforms and 3D reconstructions. It also allows easy processing of inline size-exclusion chromatography coupled SAXS data and data deconvolution using the evolving factor analysis method. It provides an alternative to closed-source programs such as Primus and ScÅtter for primary data analysis. Because it can calibrate, mask and integrate images it also provides an alternative to synchrotron beamline pipelines that scientists can install on their own computers and use both at home and at the beamline. RAW is currently used by at least four SAXS beamlines worldwide, including two in North America (the MacCHESS BioSAXS beamline at CHESS and BioCAT at the APS). It is also used at numerous home source SAXS instruments worldwide and is distributed by SAXSLAB/Xenocs with some of their SAXS instruments.

Acta Cryst. (2020). A76, a27 